\title{
Speech Signal Processing
}

語音信號處理

\author{
Berlin Chen 2003
}




\section{Course Contents}

- Both the theoretical and practical issues for spoken language processing will be considered

- Technology for Automatic Speech Recognition (ASR) will be further emphasized

- Topics to be covered

- Statistical Modeling Paradigm

- Spoken Language Structure

- Hidden Markov Models

- Speech Signal Analysis and Feature Extraction

- Acoustic and Language Modeling

- Search/Decoding Algorithms

- Systems and Applications

- Keyword Spotting, Dictation, Speaker Recognition, Spoken Dialogue, Speech Information Retrieval etc. 


\section{Textbook and References}

- Textbook:

- X. Huang, A. Acero, H. Hon, "Spoken Language Processing," Prentice Hall, 2001

- References:

- T. F. Quatieri,"Discrete-Time Speech Signal Processing Principles and Practice," Prentice Hall, 2002

- J. R. Deller, J. H. L. Hansen, J. G. Proakis, "Discrete-Time Processing of Speech Signals," IEEE Press, 2000

- F. Jelinek, "Statistical Methods for Speech Recognition," The MIT Press, 1999

- S. Young et al., "The HTK Book", Version 3.0, 2000 "http://htk.eng.cam.ac.uk"

- L. Rabiner, B.H. Juang, "Fundamentals of Speech Recognition", Prentice Hall, 1993 


\section{Grading}

- Midterm or Final: $30 \%$

- Exercises: $30 \%$

- Project: $15 \%$

- Attendance/Presentation/Report: 20\%

- Others: $5 \%$ 


\section{Introduction}

References:

1. B-H Juang and S. Furui, "Automatic Recognition and Understanding of Spoken Language - A First Step Toward Natural Human-Machine Communication," Proceedings of IEEE, August, 2000

2. I. Marsic, Member, A. Medl, And J. Flanagan, "Natural Communication with Information Systems," Proceedings of IEEE, August, 2000 


\section{Historical Review}

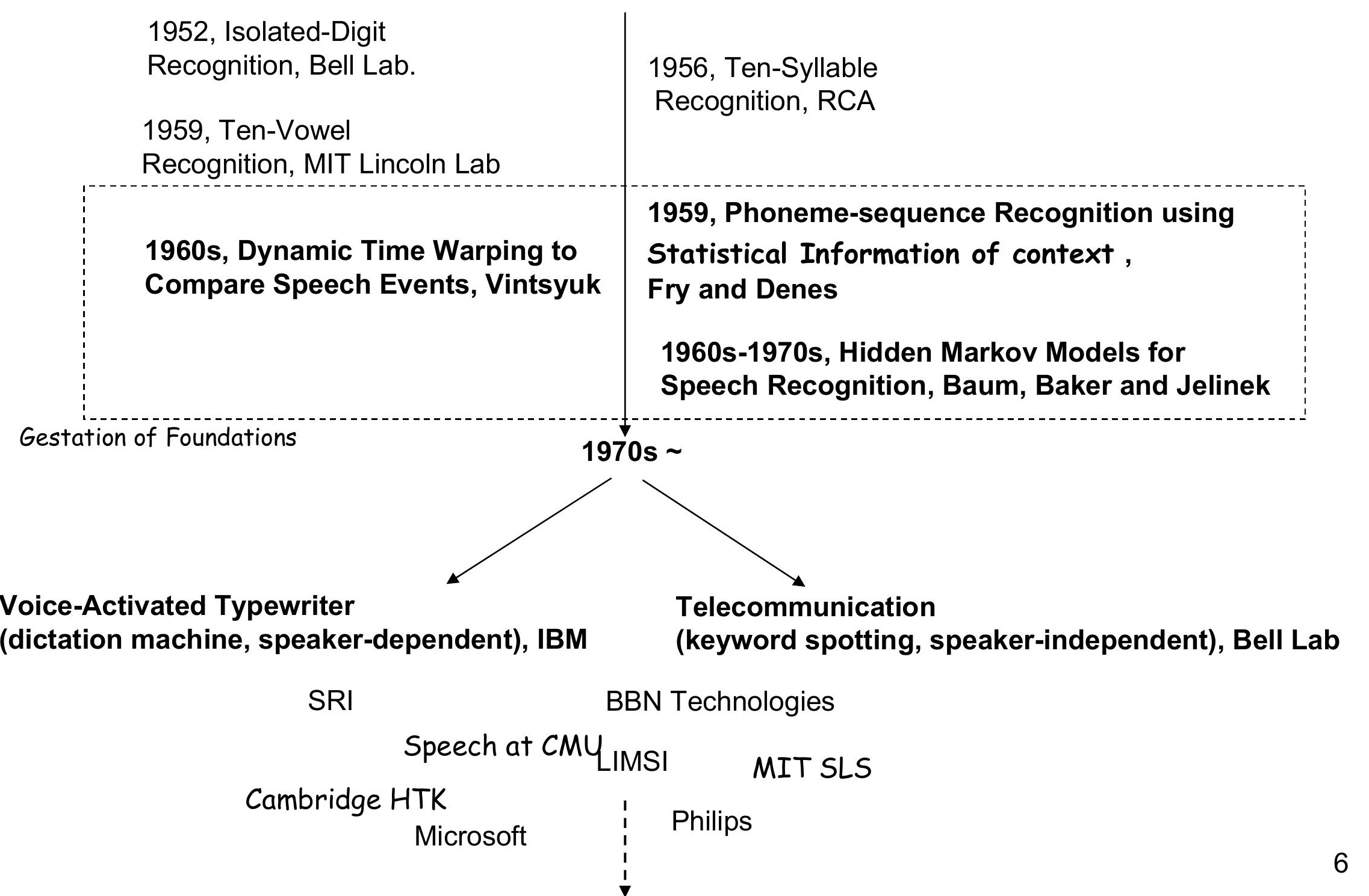




\section{Progress of Technology}

- US. National Institute of Standards and Technology (NIST)
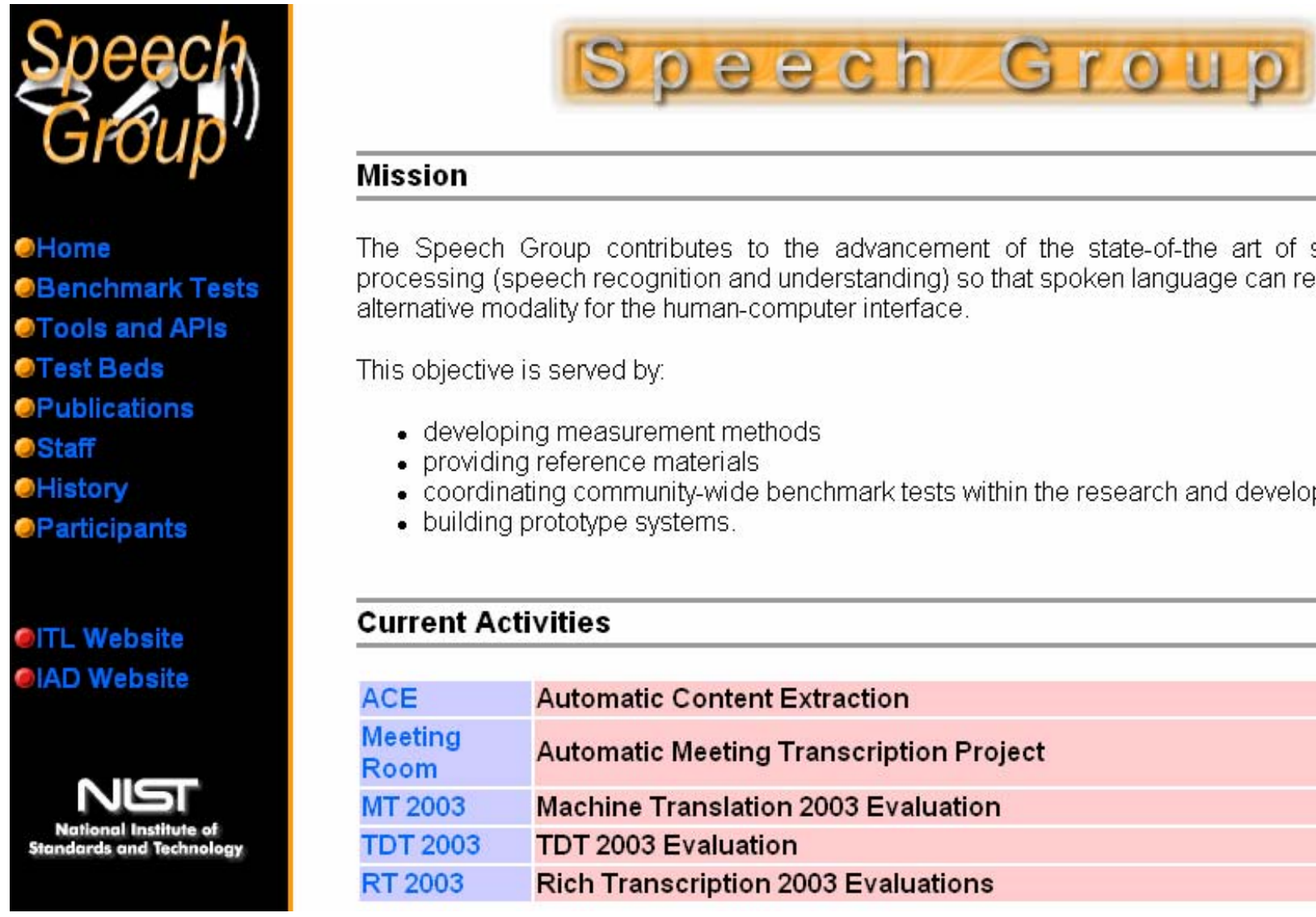

Mission

The Speech Group contributes to the advancement of the state-of-the art of spoken language processing (speech recognition and understanding) so that spoken language can reliably serve as an alternative modality for the human-computer interface.

This objective is served by:

- developing measurement methods

- providing reference materials

- coordinating community-wide benchmark tests within the research and development community

- building prototype systems.

\section{Current Activities}

\begin{tabular}{ll} 
ACE & Automatic Content Extraction \\
$\begin{array}{l}\text { Meeting } \\
\text { Room }\end{array}$ & Automatic Meeting Transcription Project \\
\hline MT 2003 & Machine Translation 2003 Evaluation \\
\hline TDT 2003 & TDT 2003 Evaluation \\
\hline RT 2003 & Rich Transcription 2003 Evaluations \\
\hline
\end{tabular}

http://www.nist.gov/speech/ 


\section{Progress of Technology}

- Generic Application Areas (vocabulary vs. speaking style)

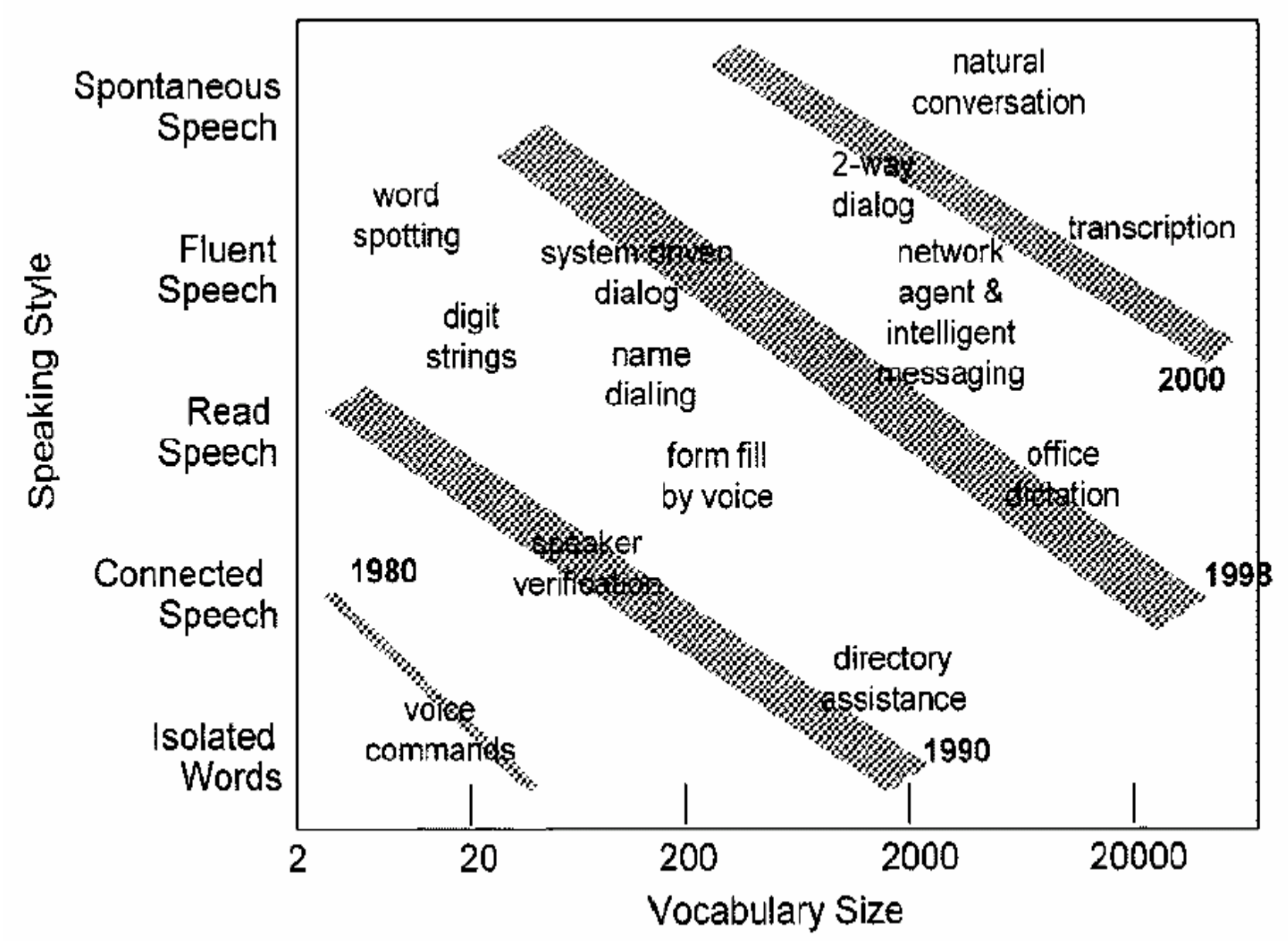




\section{Progress of Technology}

- Benchmarks of ASR performance: Overview

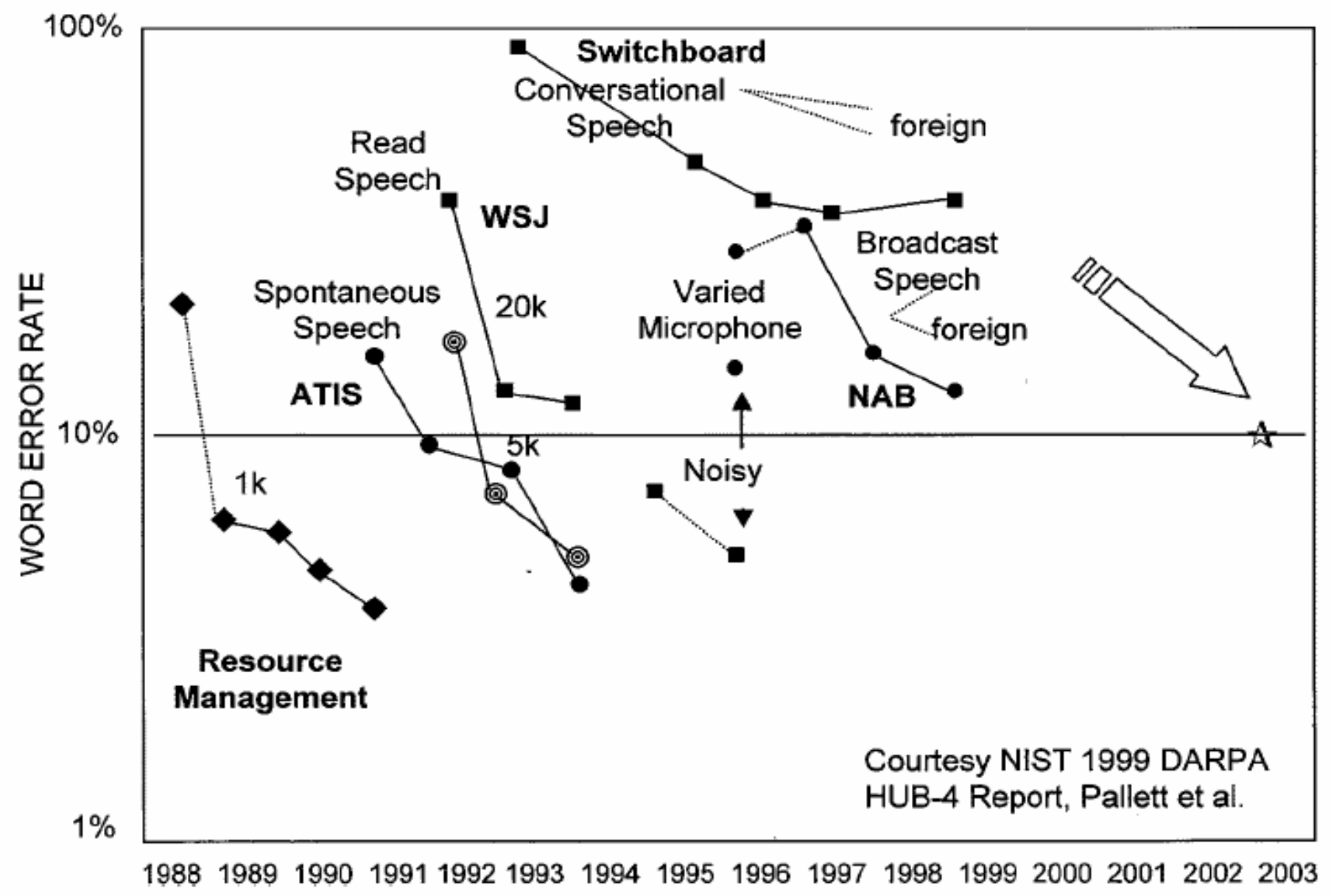




\section{Progress of Technology}

- Benchmarks of ASR performance: Broadcast News Speech

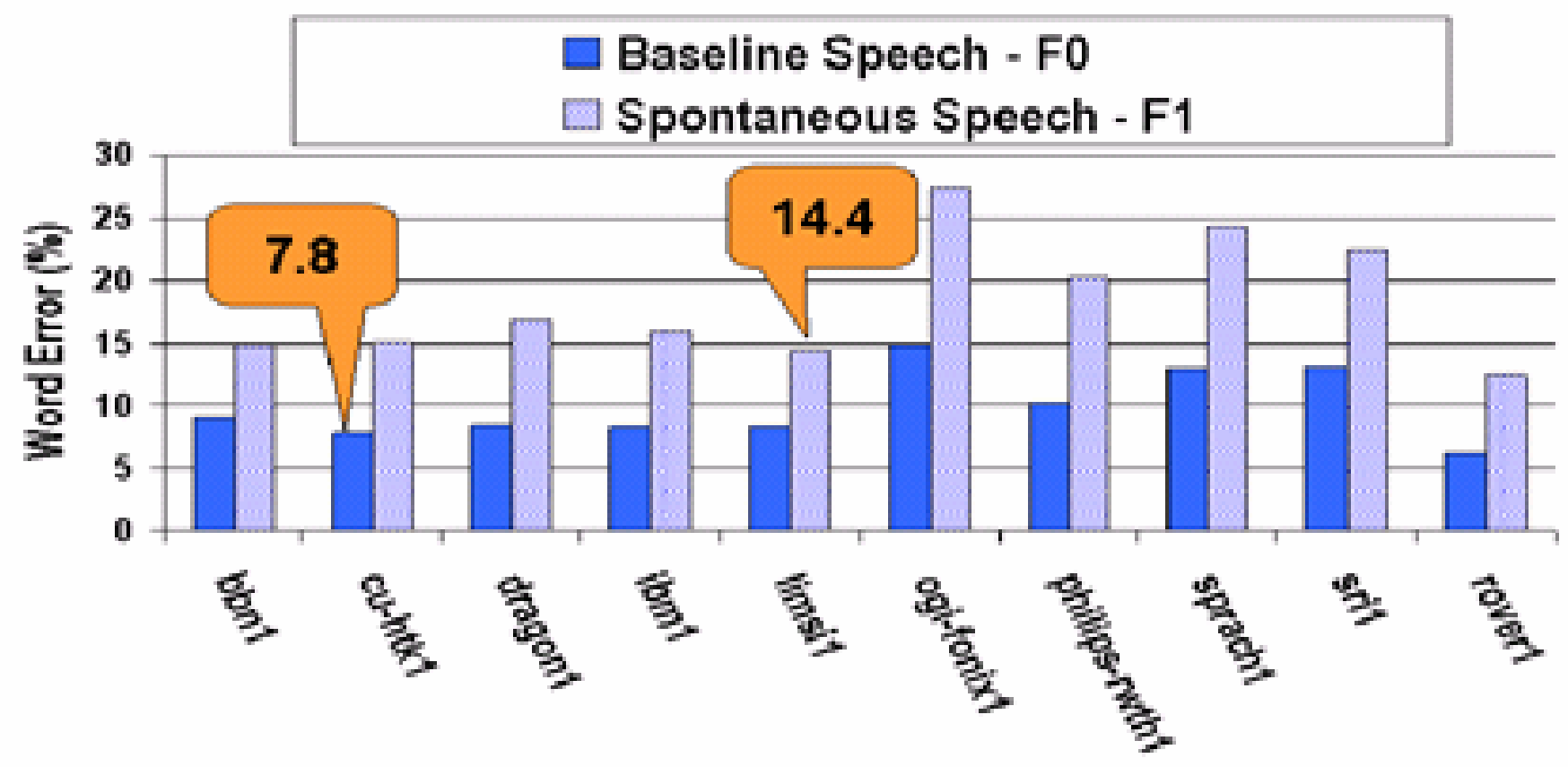




\section{Determinants of Speech Communication}

Speech Generation

Speech Understanding

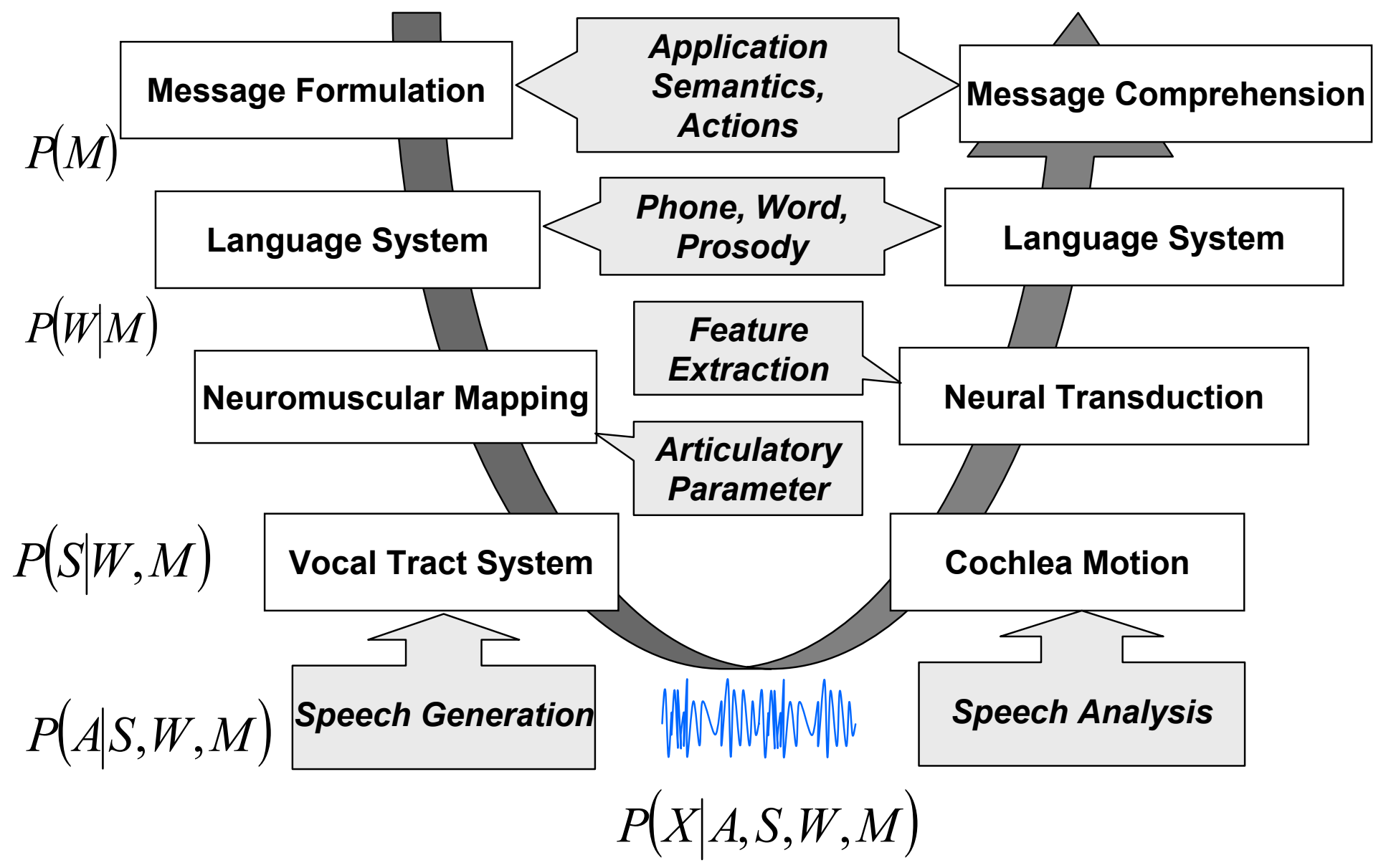




\section{Statistical Modeling Paradigm}

- The statistical modeling paradigm used in speech and language processing

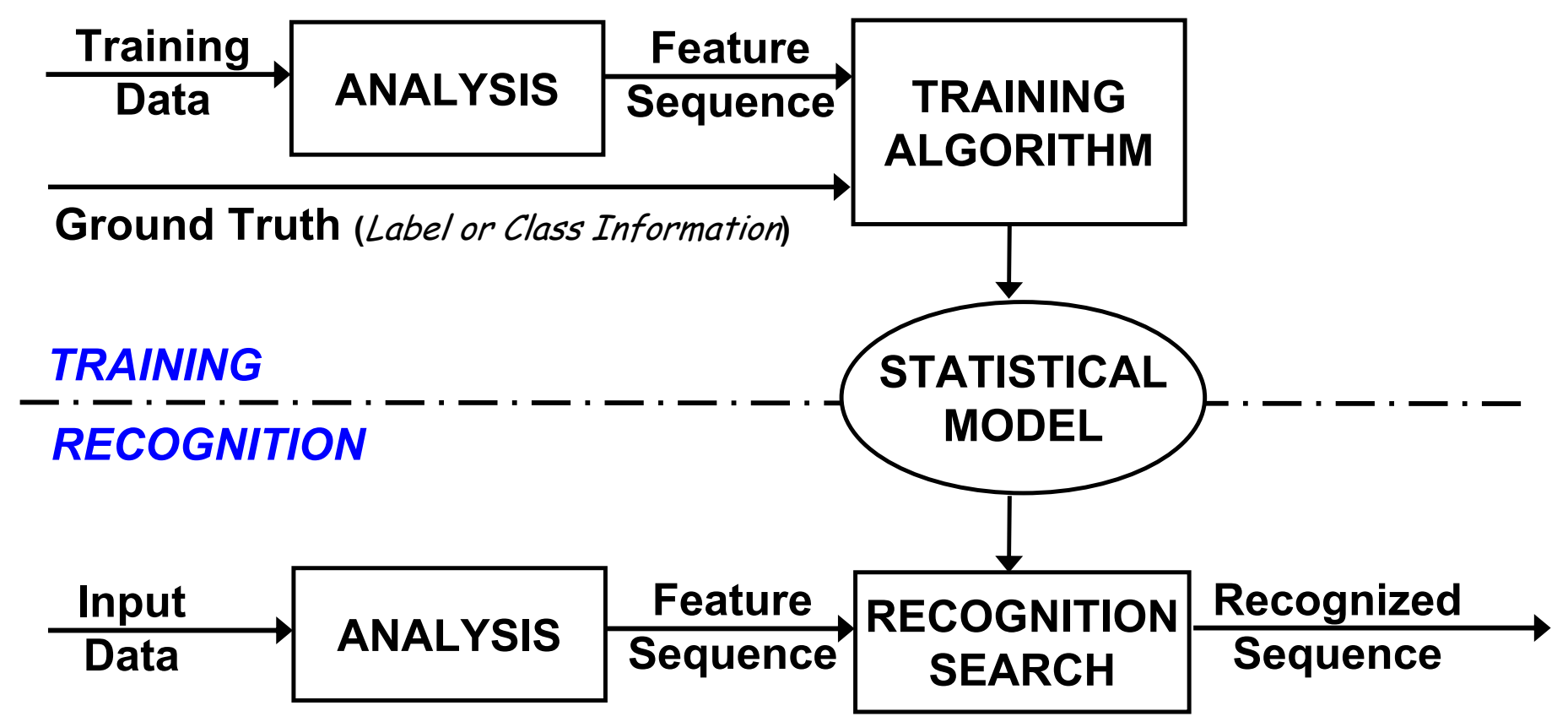




\section{Statistical Modeling Paradigm}

- Approaches based on Hidden Markov Models (HMMs) dominate the area of speech recognition

- HMMs are based on rigorous mathematical theory built on several decades of mathematical results developed in other fields

- HMMs are generated by the process of training on a large corpus of real speech data

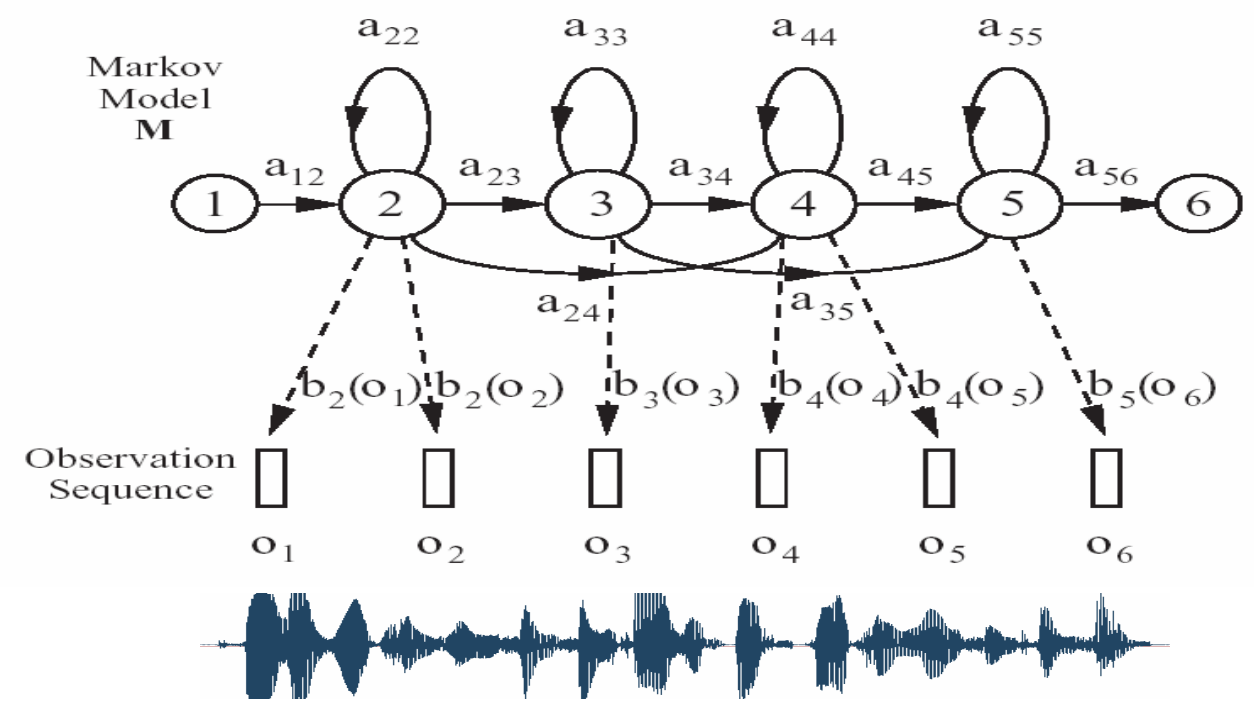




\section{Difficulties: Speech Variability}

\section{Pronunciation}

Variation

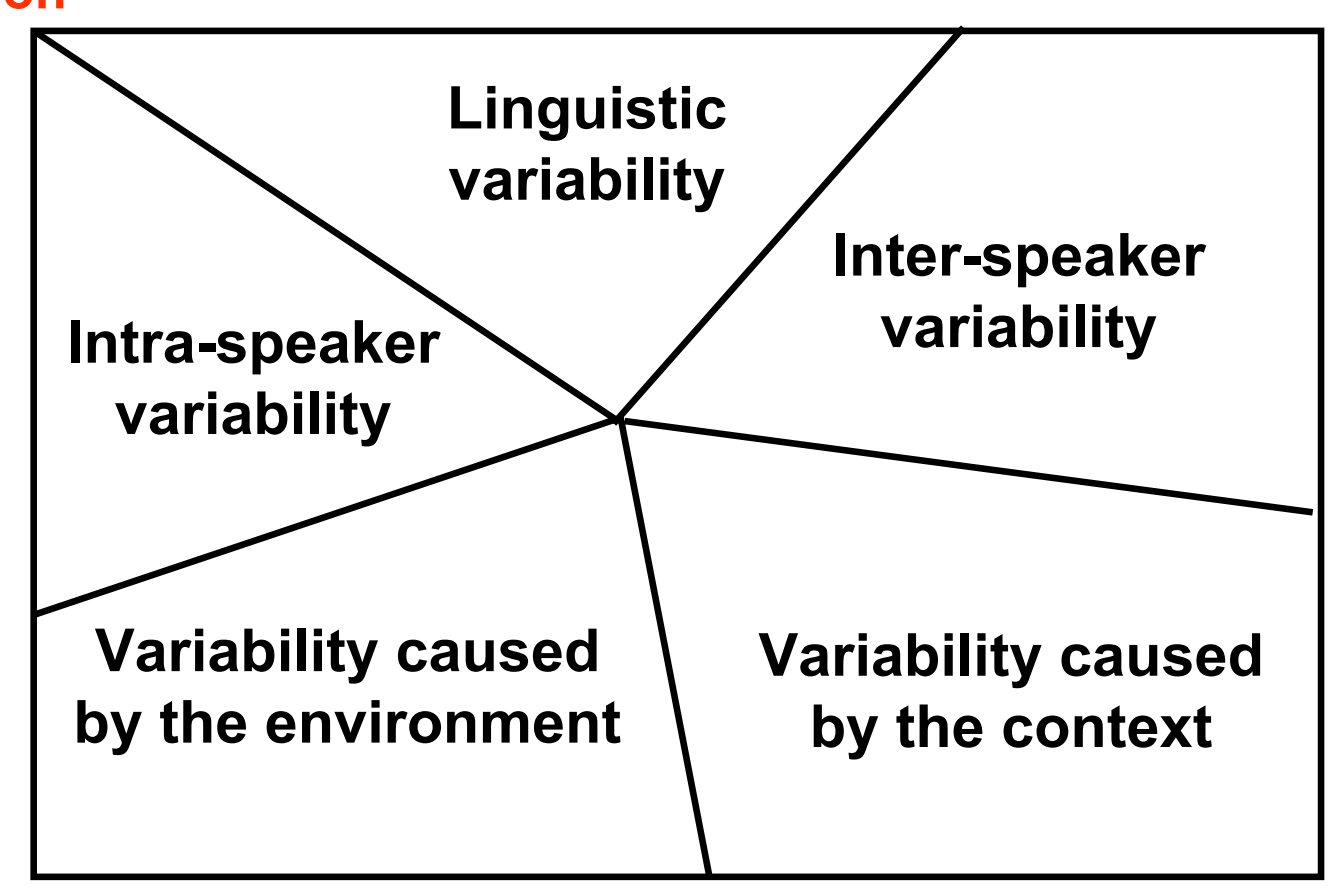




\section{Large Vocabulary Continuous Speech Recognition}

語音特徵參數抽取

語言解碼/搜寻演算法

語音輸入

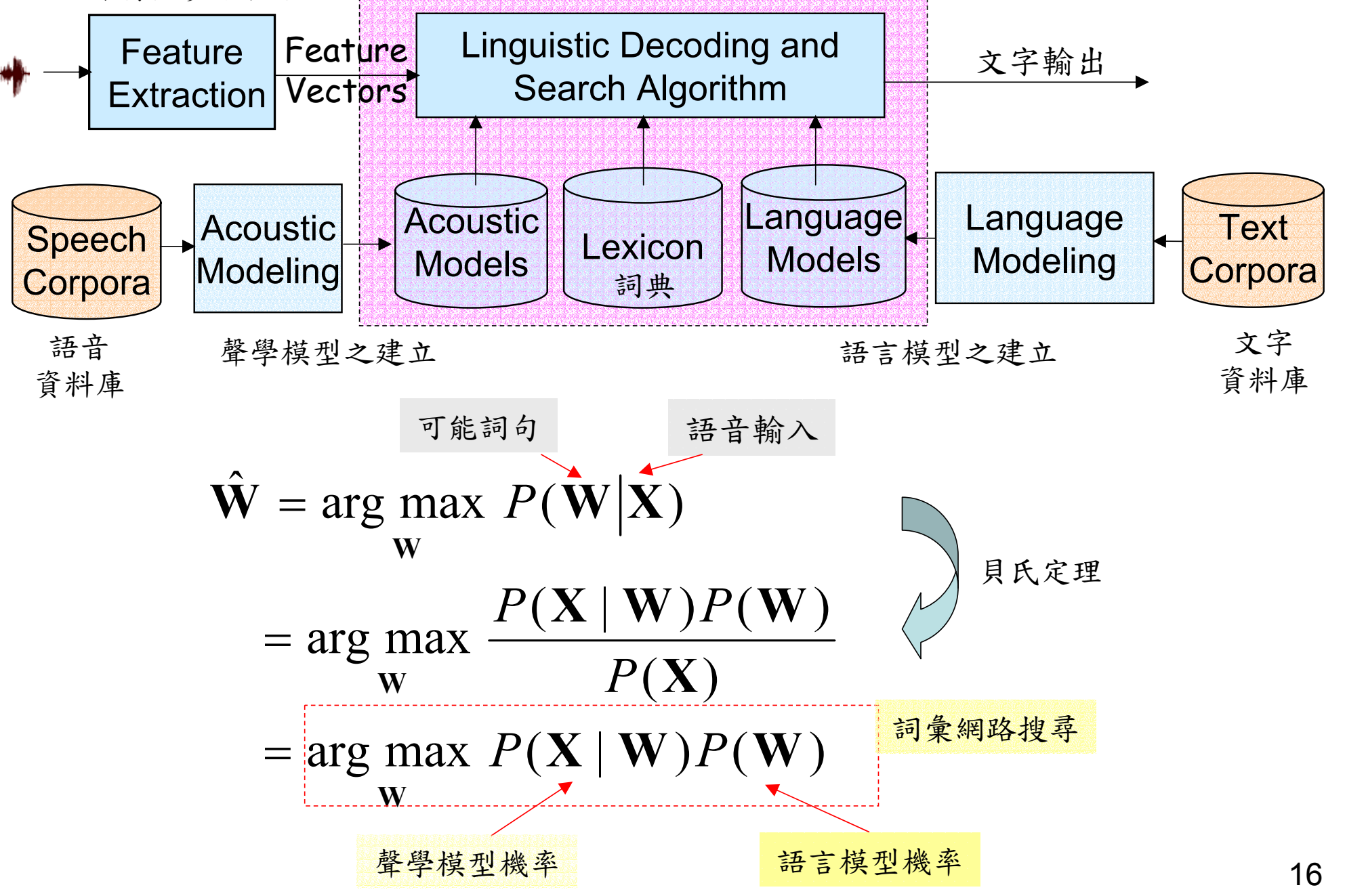




\section{Large Vocabulary Continuous Speech Recognition}

\section{- Transcription of Broadcast News Speech}

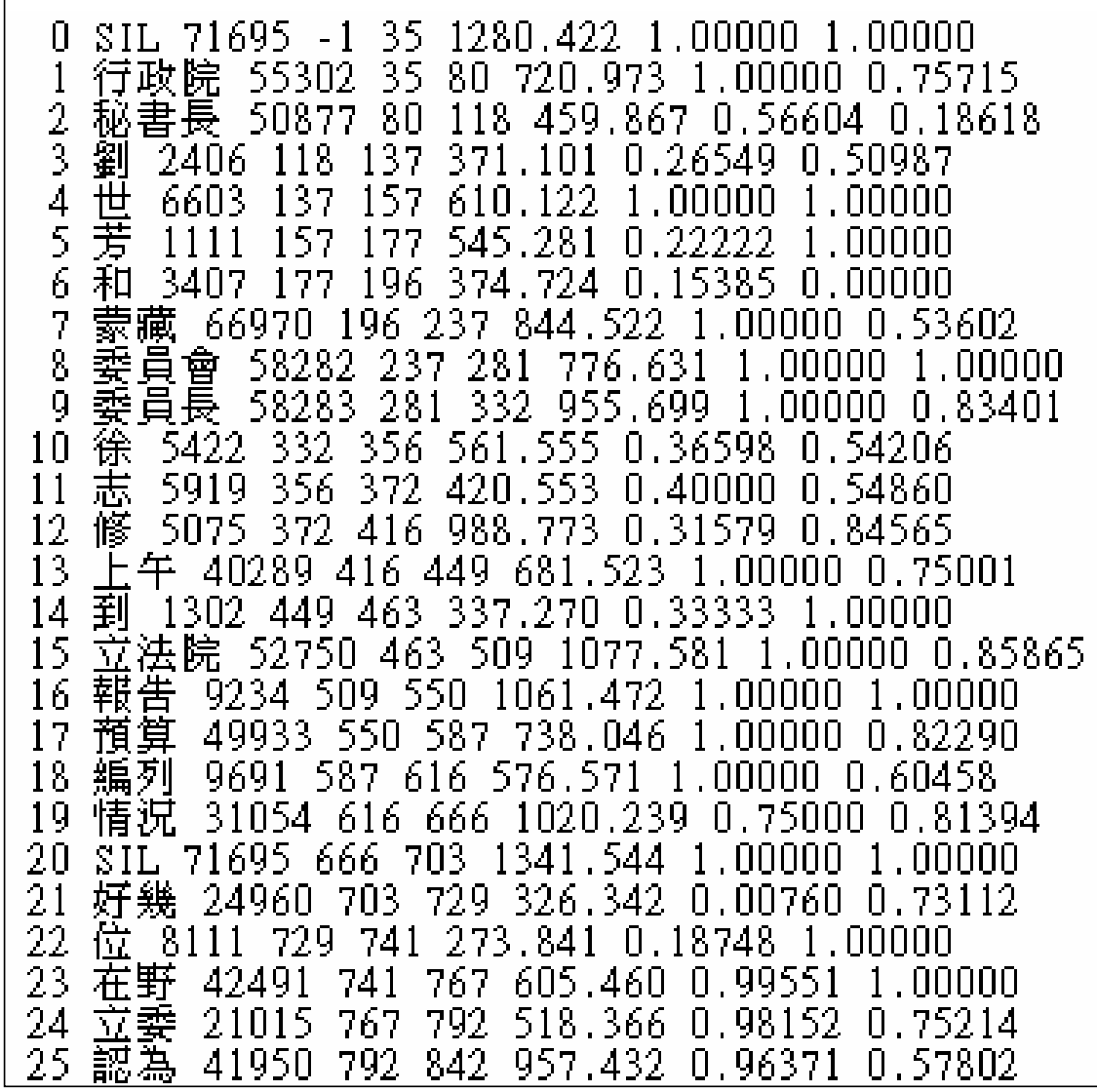

0 SIL $71695 \quad-1 \quad 35 \quad 1280.422 \quad 1.000001,00000$

1 行政院 $553023580720.973 \quad 1$ ，00000 0.75715

2 柲晋長 $5087780118459.867 \quad 0.56604 \quad 0.18618$

3 紫 $2406 \quad 118 \quad 137 \quad 371.101 \quad 0.26549 \quad 0.50987$

4 世 $6603137 \quad 157 \quad 610.122 \quad 1.000001 .00000$

5 苦 $1111157 \quad 177 \quad 545.281 \quad 0.22222 \quad 1$.00000

6 和 $3407 \begin{array}{llllll}177 & 196 & 374.724 & 0.15385 & 0.00000\end{array}$

7 菜藏 $66970196237844.522 \quad 1$, 000000.53602

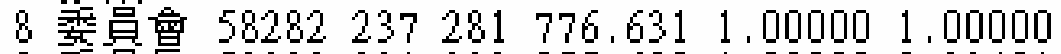

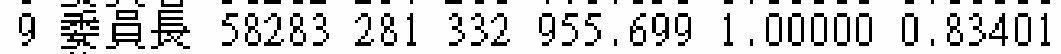

10 保 $5422 \quad 332 \quad 356 \quad 561.555 \quad 0.36598 \quad 0.54206$

11 志 $5919356 \quad 372420.553 \quad 0.400000 .54860$

12 梆 $5075 \quad 372416 \quad 988.773 \quad 0.31579 \quad 0.84565$

13 上午 40289416449681.5231 . 00000 0.75001

14 到 $1302 \quad 449463 \quad 337.270 \quad 0.33333 \quad 1$, 00000

15 妾法院 $5275046350907077.581 \quad 1$, 000000.85865

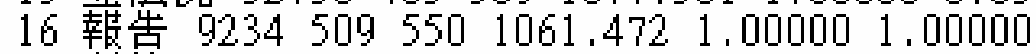

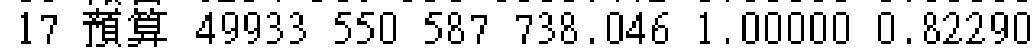

18 新扁列 $9691587616576.571 \quad 1.000000 .60458$

19 情㫛 $31054 \quad 616 \quad 666 \quad 1020.239 \quad 0.75000 \quad 0.81394$

20 SIL $71695666703 \quad 1341.544 \quad 1$.00000 1 .00000

21 对幽 $24960703729326,342 \quad 0.00760 \quad 0.73112$

22 偣 $8111 \quad 729741273,841 \quad 0.18748 \quad 1$, 00000

23 在野 $42491741767605.460 \quad 0.99551 \quad 1$ ，00000

24 音琹 $21015 \quad 767 \quad 792 \quad 518.366 \quad 0.98152 \quad 0.75214$

25 諗為 $41950792842 \quad 957.432 \quad 0.96371 \quad 0.57802$

26 SIL $71695 \quad 842 \quad 872 \quad 1138.477 \quad 1.000001 .00000$ 27 行政院 $55302 \quad 872 \quad 934 \quad 1120.105 \quad 0.86107 \quad 0.87346$

28 磨然 $29583934971804.2590 .86107 \quad 0.95910$

29 不 $369971988288.7280 .69917 \quad 1.00000$

30 事認 $38027988 \quad 1043 \quad 931.888 \quad 0.46961 \quad 0.40323$

31 外莐 $47896 \quad 1043 \quad 1084 \quad 786.448 \quad 1$. 000001 1.00000

32 為 $8063 \quad 1084 \quad 1100316.677 \quad 0.30057 \quad 1.00000$

33 我國 $47848 \quad 11001135804.705 \quad 1$. 10000 1 .00000

34 領圭 $20696 \quad 1135 \quad 1186 \quad 778.006 \quad 0.76186 \quad 0.96218$

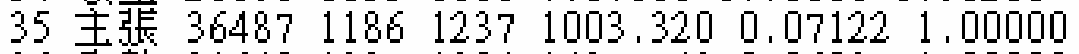

36 圣數 $316491237 \quad 1304 \quad 1427,742 \quad 0.06937 \quad 1$ ，00000

37 刪搼 $39728 \quad 1304 \quad 1349818.702 \quad 1$.00000 0.65401

38 暴藏 $66970 \quad 1349 \quad 1392 \quad 790.226 \quad 0.00928 \quad 0.51333$

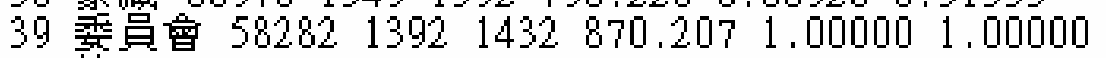

40 的 $1269 \quad 1432 \quad 1441 \quad 165.007 \quad 0.16667 \quad 1.00000$

41 預算 $499331441 \quad 1490 \quad 1304.056 \quad 0.23077 \quad 1$. 00000

42 SIL $7169514901522 \quad 1101.7601$.00000 1 .00000

43 佂事 $43981 \quad 1522 \quad 1566 \quad 1100.780 \quad 0.05556 \quad 0.76556$

44 過 $3023 \quad 1566 \quad 1580 \quad 279.248 \quad 0.07692 \quad 1$. 00000

45 院長 $49392 \quad 1580 \quad 1613 \quad 632,123 \quad 0.10656 \quad 0.80456$

46 許 $3809 \quad 1613 \quad 1634 \quad 526.977 \quad 0.083331$.00000

47 志 $59191634 \quad 1650222.692 \quad 0.052631$. 00000

$\begin{array}{lllllll}48 & \text { 䧸 } 5420 & 1650 & 1685 & 762.830 & 0.33333 & 0.56287\end{array}$

$\begin{array}{llllllll}49 & +\mathrm{t} & 7545 & 1685 & 1706 & 484.241 & 0.18462 & 1.00000\end{array}$

50 䓇 $2847 \quad 1706 \quad 1721 \quad 403.345 \quad 0.18182 \quad 1.00000$

51 下参 $320601721 \quad 1781 \quad 1458.783 \quad 0.06522 \quad 1$.00000 52 SIL $71695 \quad 1781 \quad 18432489.860 \quad 1$. 000001 . 000000 


\section{Spoken Dialogue}

- Spoken language is attractive because it is the most natural, convenient and inexpensive means of exchanging information for humans

- In mobilizing situations, using keystrokes and mouse clicks could be impractical for rapid information access through small handheld devices like PDAs, cellular phones, etc. 


\section{Spoken Dialogue}

- Flowchart

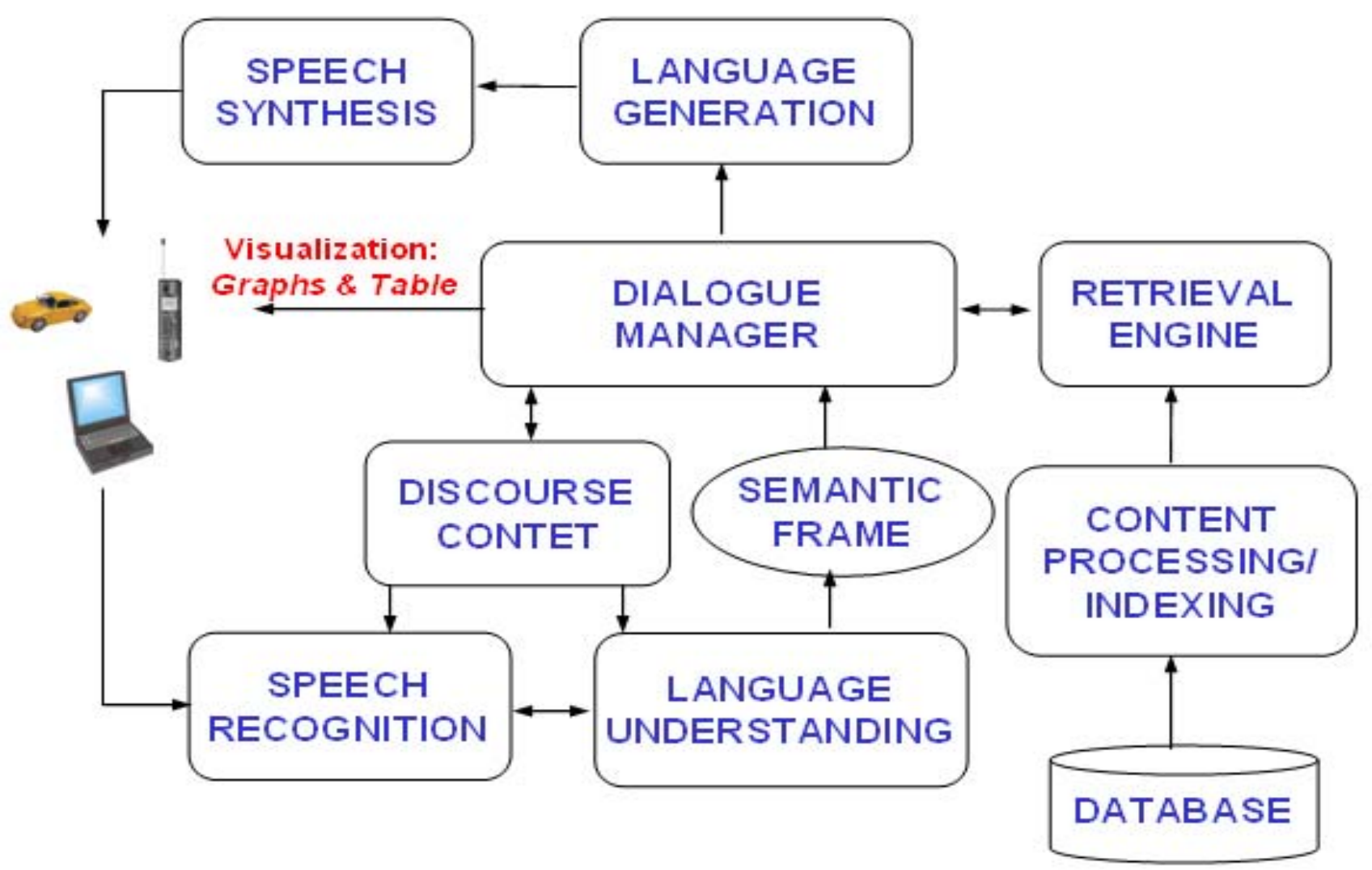




\section{Spoken Dialogue}

- Multimodality of Input and Output

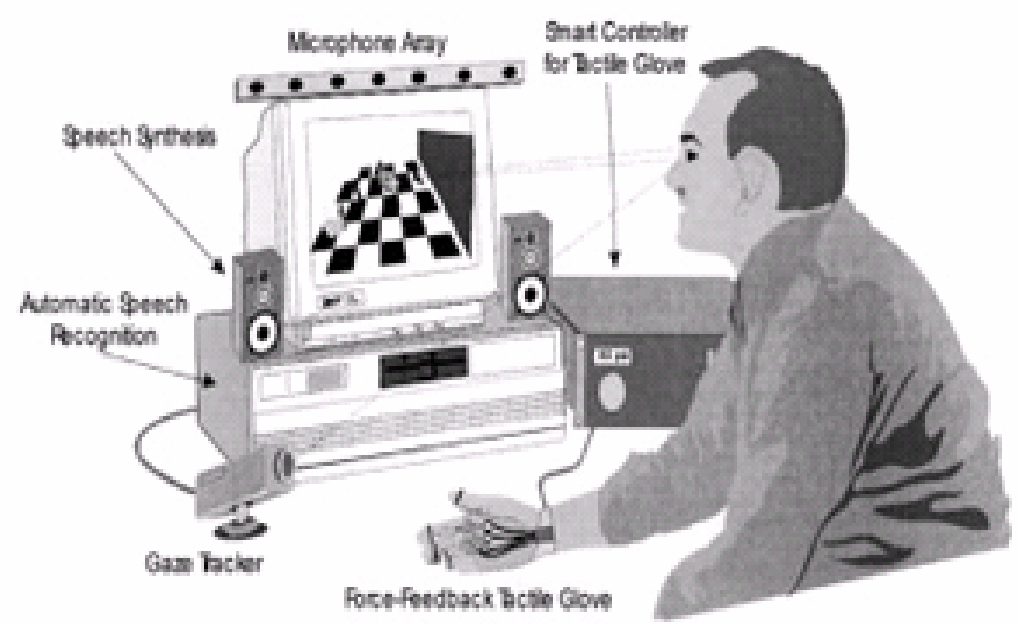

Experimental client workstation incorporating sight, sound, and touch modalities for human/machine communication. The eye tracker provides a gaze-controlled cursor for indicating objects in the display. The tactile force-feedback glove allows displayed objects to be grasped, "felt," and moved. Hands-free speech recognition and synthesis provides natural conversational interaction [7]. 


\section{Spoken Dialogue}

- Deployed Dialogue Systems

\begin{tabular}{l||c|c|c|c}
\hline Domain & Language & Vocabulary & \multicolumn{2}{|c}{ Average } \\
& & Size & Words/Utt & Utts/Dialogue \\
\hline CSELT Train Timetable Info & Italian & 760 & 1.6 & 6.6 \\
SpeechWorks Air Travel Reservation & English & 1000 & 1.9 & 10.6 \\
Philips Train Timetable Info & German & 1850 & 2.7 & 7.0 \\
\hline CMU Movie Information & English & 757 & 3.5 & 9.2 \\
CMU Air Travel Reservation & English & 2851 & 3.6 & 12.0 \\
LIMSI Train Timetable Info & French & 1800 & 4.4 & 14.6 \\
MIT Weather Information & English & 1963 & 5.2 & 5.6 \\
MIT Air Travel Reservation & English & 1100 & 5.3 & 14.1 \\
AT\&T Operator Assistance & English & 4000 & 7.0 & 3.0 \\
\hline Air Travel Reservations (human) & English & $?$ & 8.0 & 27.5 \\
\hline
\end{tabular}




\section{Spoken Dialogue}

- Topics vs. Dialogue Terms

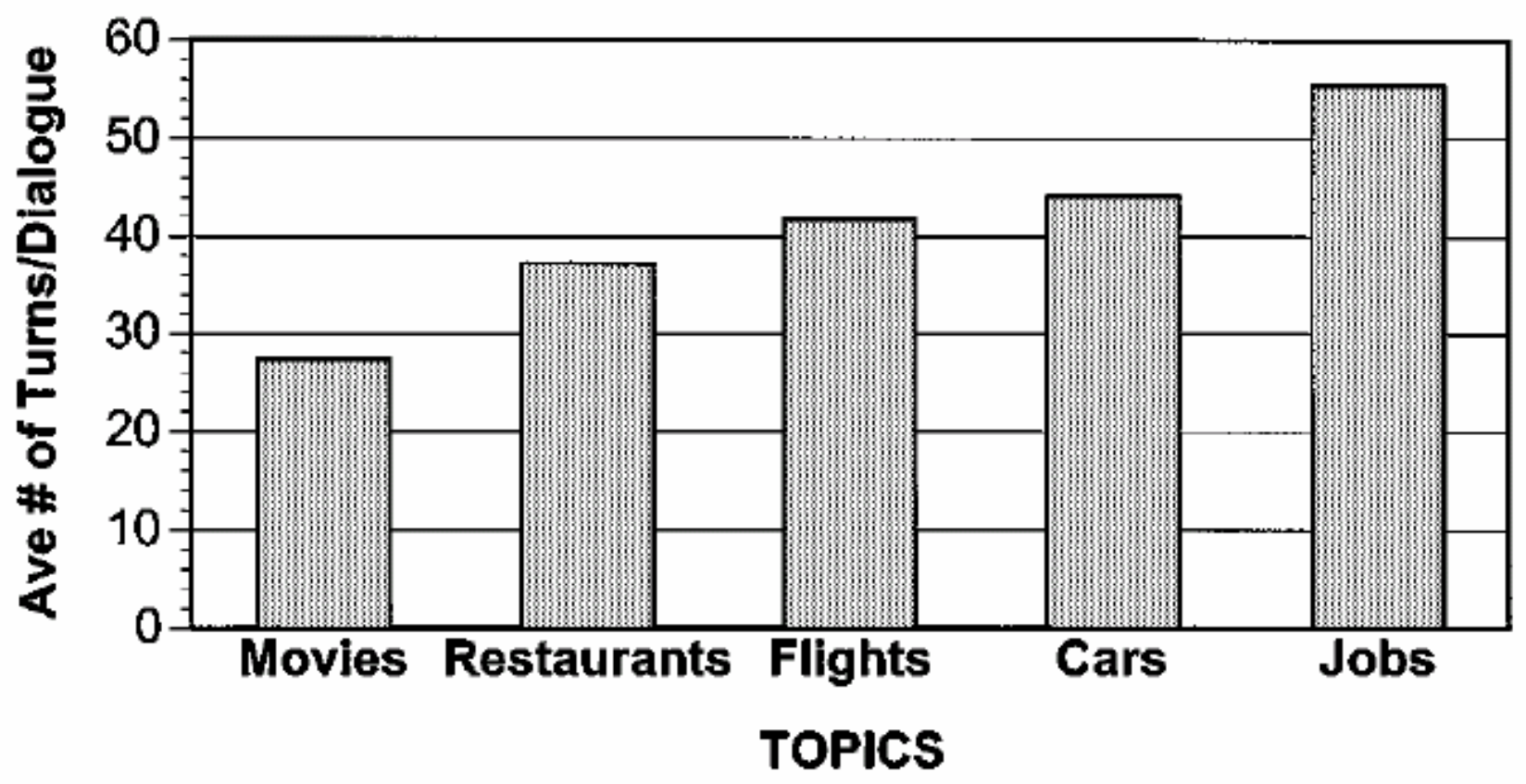




\section{Speech-based Information Retrieval}

- Task :

- Automatically indexing a collection of spoken documents with speech recognition techniques

- Retrieving relevant documents in response to a text/speech query

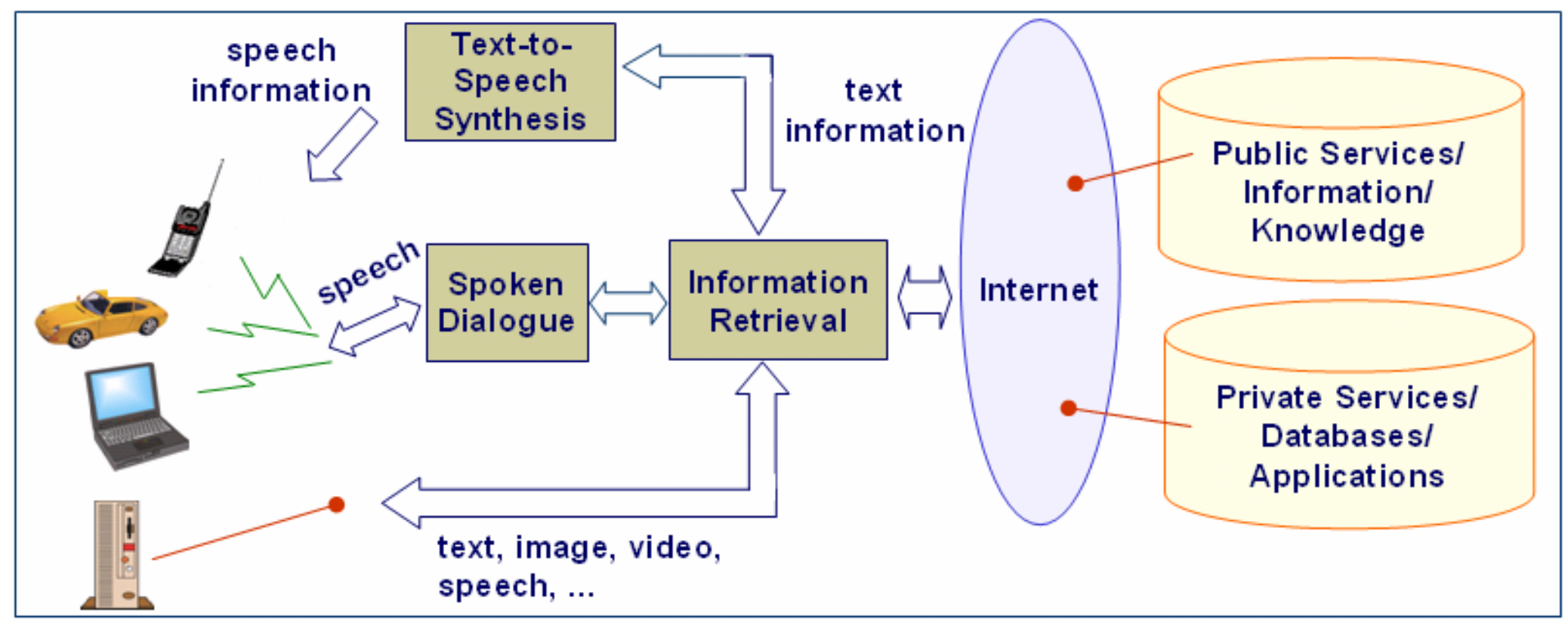




\section{Speech-based Information Retrieval (cont.)}

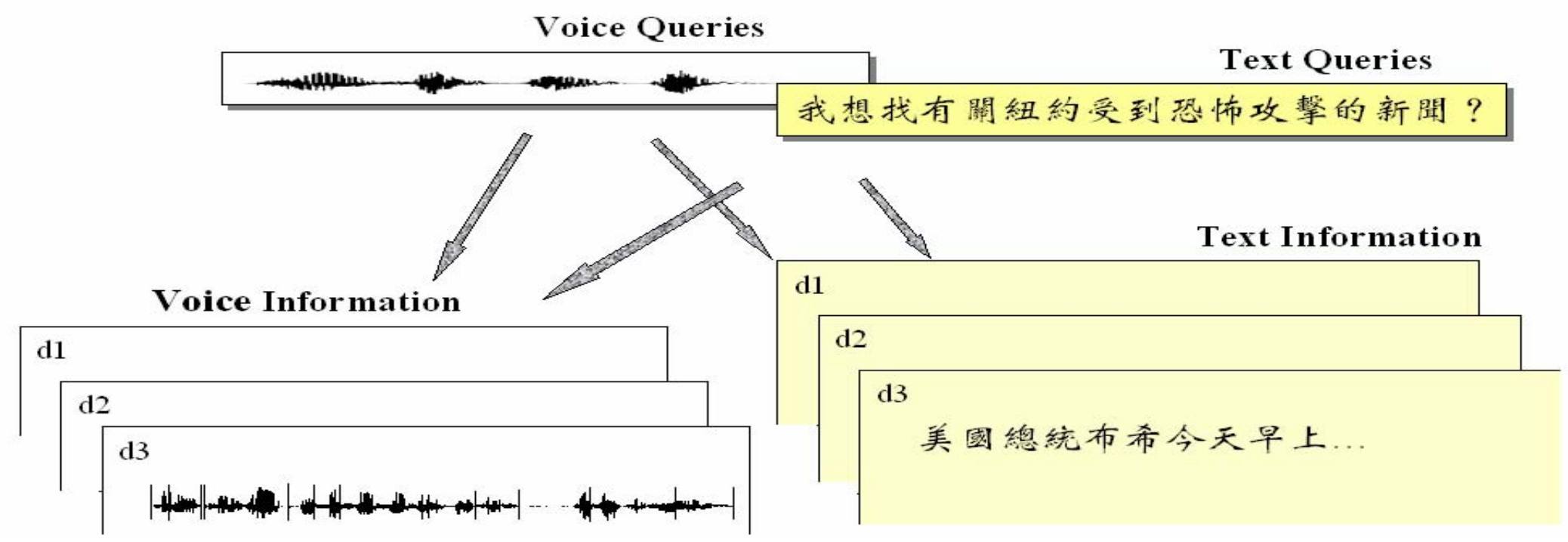

在四種不同時機下的資訊檢索過程。使用聲音問句(VQ, Voice Queries)或文字問句(TQ, Text Queries)去檢索聲音資訊(VI, Voice Information)或者是傳統的文字資訊(TI, Text Information)。 


\section{Speech-based Information Retrieval (cont.)}

翰入聲音問句：“請幫我查總统府升旗典䀅”

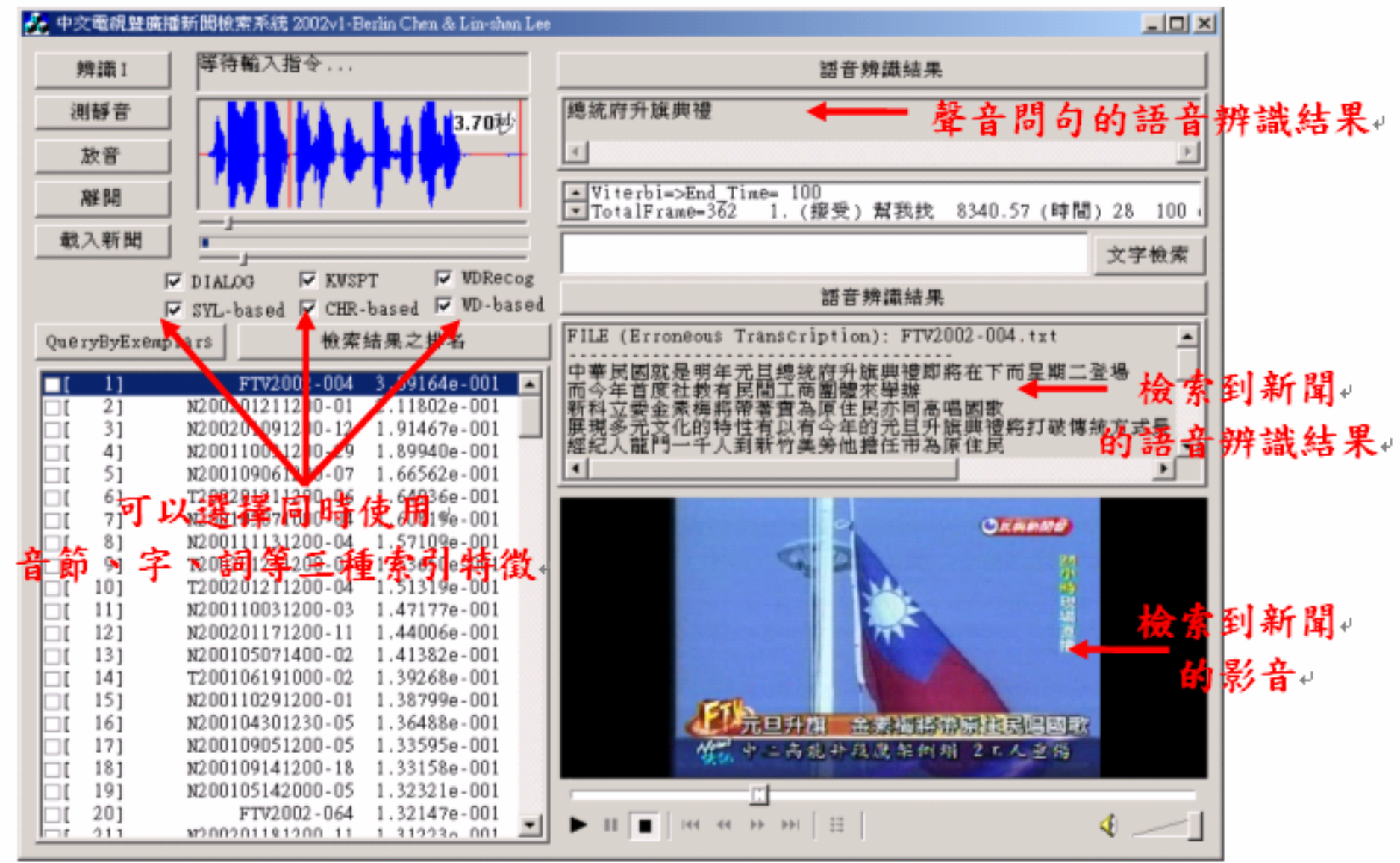




\section{Speech-based Information Retrieval (cont.)}
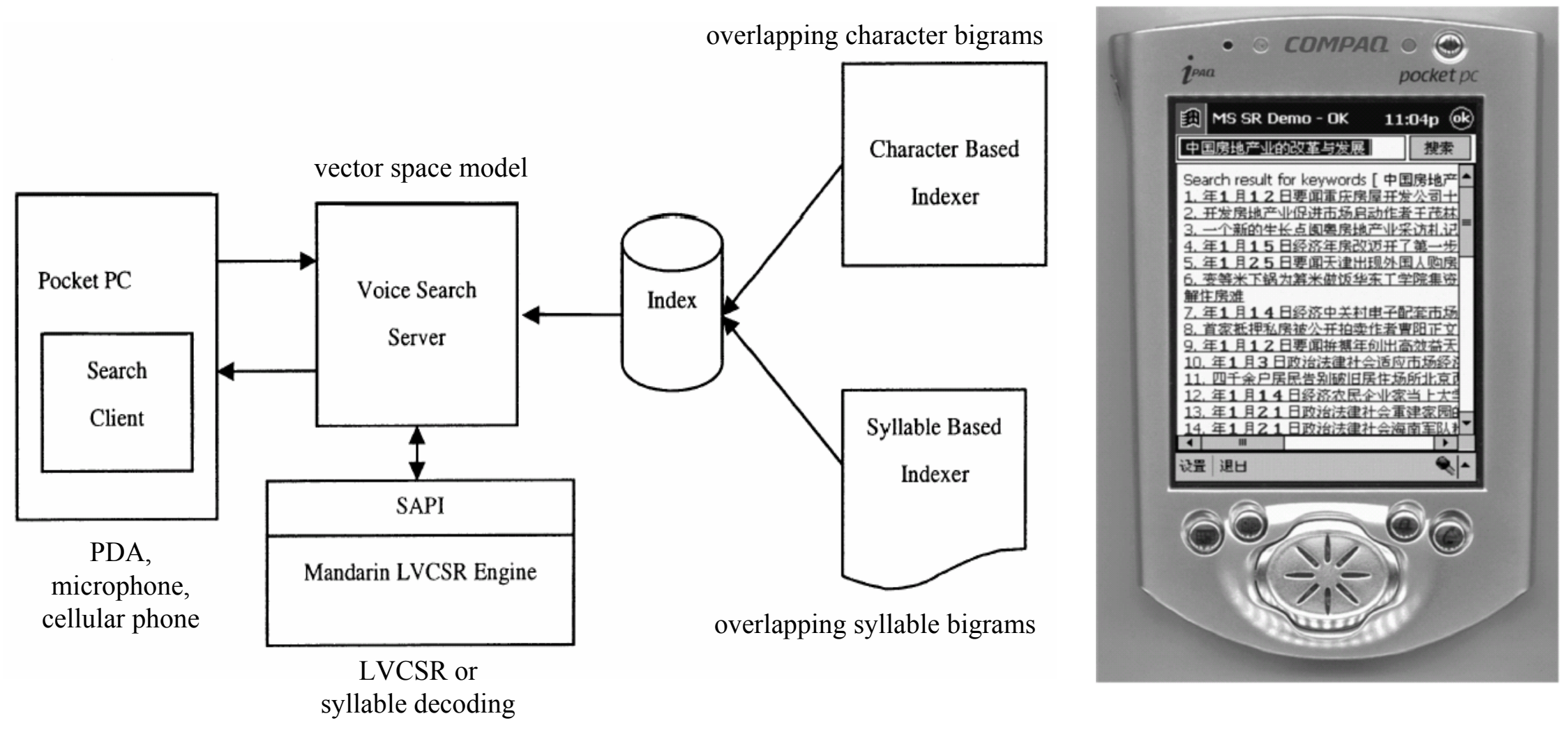


\section{Applications}

Applied

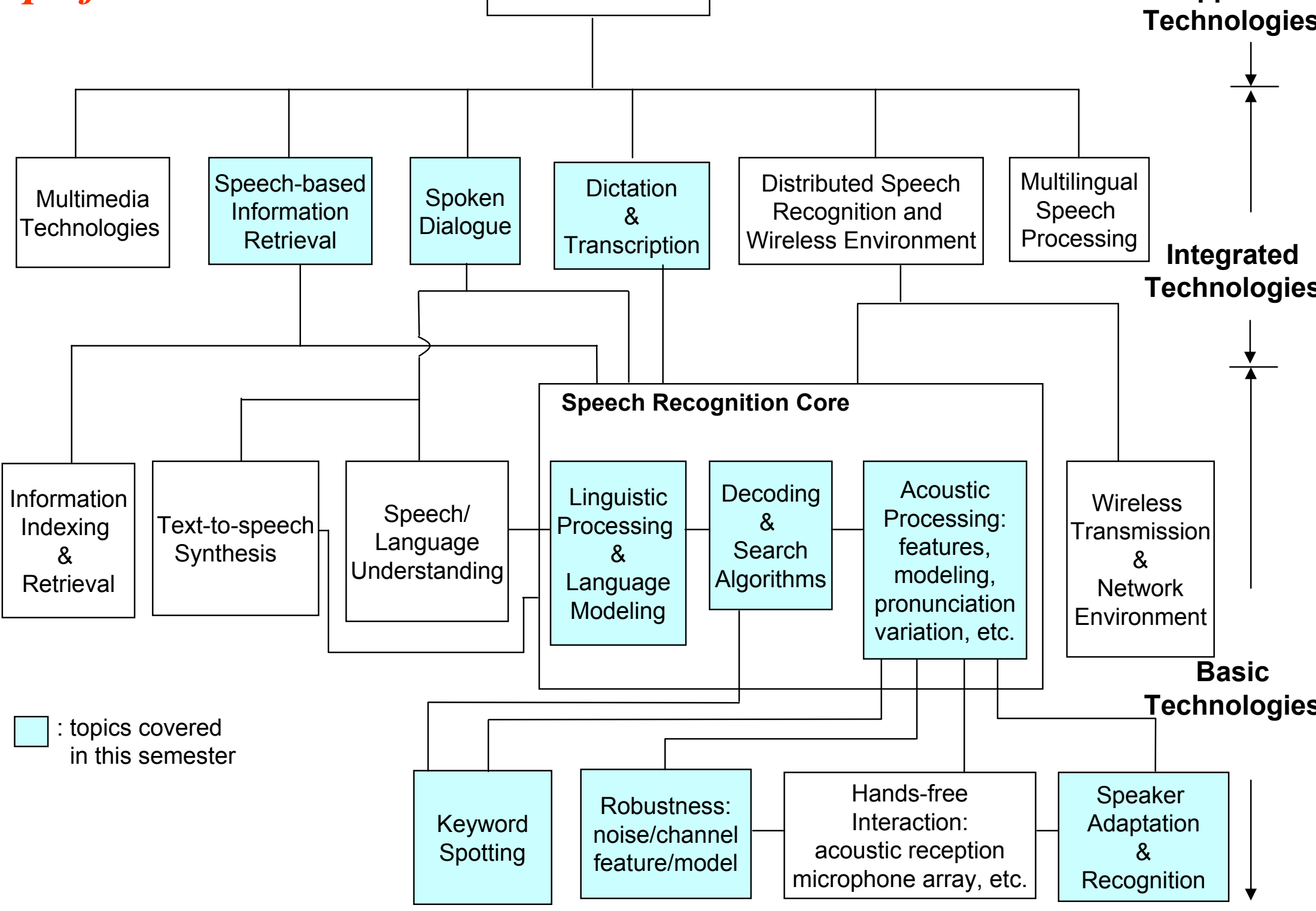




\section{Speech Processing Toolkit}

- HTK (Hidden Markov Model ToolKit)

- A toolkit for building Hidden Markov Models (HMMs)

- The HMM can be used to model any time series and the core of HTK is similarly general-purpose

- In particular, for the acoustic feature extraction, HMMbased acoustic model training and HMM network decoding 


\section{Speech Processing Toolkit}

- HTK (Hidden Markov Model ToolKit)

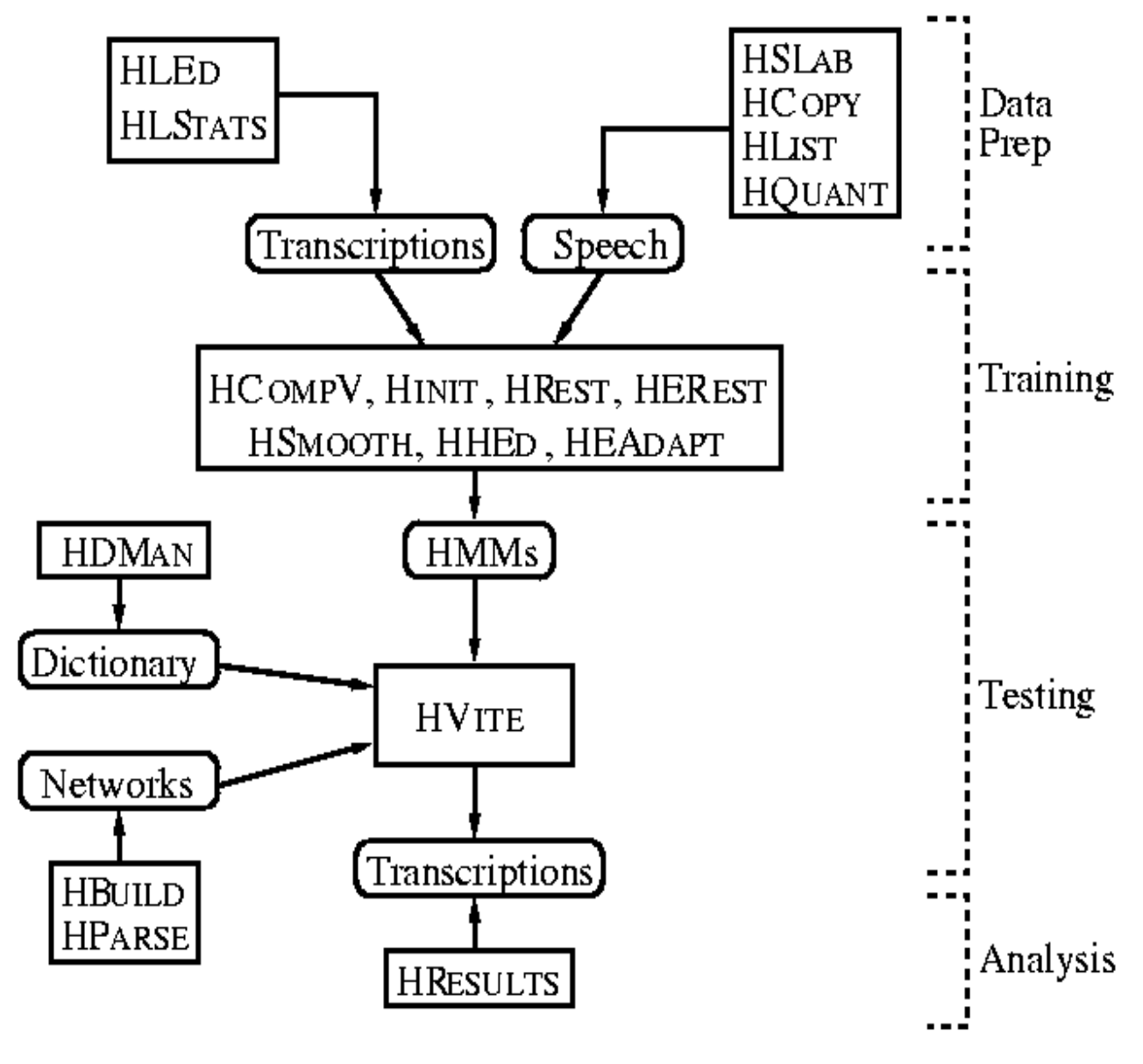




\section{Speech Industry}

- Telecommunication

- Information Appliance

- Interactive Voice Response

- Voice Portal

- Multimedia Database

- Education
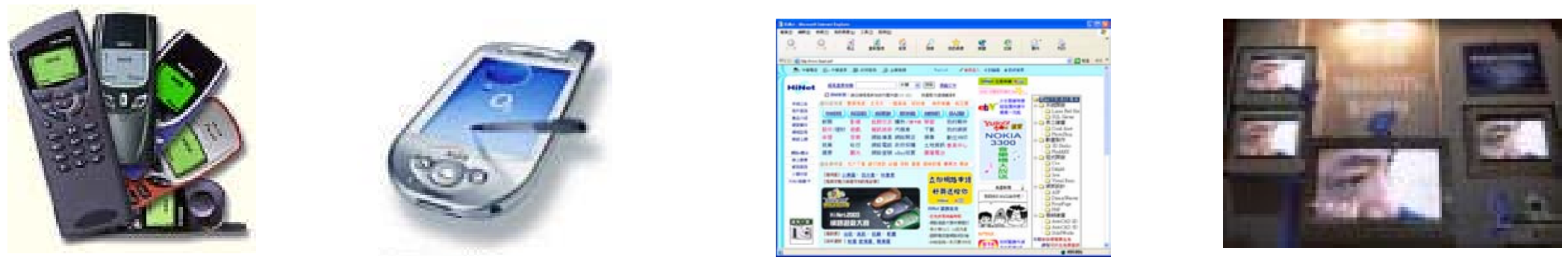


\section{Speech Workshop}

- Date: 19 September, 2003

- Place: National Tsing Hua Univ., Hsinchu

- Webpage: http://140.114.75.26/rocling/

\begin{tabular}{|c|c|c|}
\hline 日期 & 時間 & 會講內容 \\
\hline \multirow{4}{*}{ 9/19 } & $13: 30 \sim 14: 20$ & 演講：當前語音技術研究之邆勢與展望 \\
\hline & $14: 30 \sim 15: 20$ & 演講：車內環境之對話系統 \\
\hline & $15: 40 \sim 16: 20$ & 坐談會：電信學門語音處理領域墢展與國際合作 \\
\hline & \multicolumn{2}{|c|}{ 註：13:30 17:00國科會研究計畫成果墢表(壁報) } \\
\hline
\end{tabular}

